\title{
Refractory Tamponade: Use of Ultrasound Contrast Agent to Distinguish Aortic Dissection from Cardiorrhexis
}

Joel A Garcia ${ }^{1,2}$ and Mori J Krantz ${ }^{1,2 *}$

${ }^{1}$ Department of Medicine, Division of Cardiology, University of Colorado Hospital and Health Sciences Center, Denver, CO, USA

${ }^{2}$ Department of Medicine, Division of Cardiology, Denver Health Medical Center, Denver, CO, USA

\begin{abstract}
We report a case of a ruptured proximal aortic dissection with cardiac tamponade, which masqueraded as myocardial rupture or cardiorrhexis. An 84-year-old female had sudden onset of chest pain with loss of consciousness and a mechanical fall. Echocardiography demonstrated pericardial effusion with hematoma overlying the apicallateral wall and an echo free space suggestive of contained, ventricular free-wall rupture. However, intravenous injection of ultrasound contrast agent revealed no flow into the pericardial space excluding cardiorrhexis. Refractory hemodynamic compromise required an emergency pericardial window, but the patient remained unstable. Surgical exploration revealed a Debakey Type-A aortic dissection with communication into the pericardial space. To our knowledge, this is the first description of the use of ultrasound contrast microbubbles as a diagnostic strategy to exclude cardiorrhexis. The impact of tamponade treatment as the result of aortic dissections and the diagnostic challenge in differentiating it from other catastrophic causes of tamponade are reviewed.
\end{abstract}

Keywords: Cardiac tamponade; Aortic dissection; Pericardiocentesis; Surgical exploration; Echocardiography

\section{Introduction}

The pericardium consists of a two-layered sac that surrounds the heart, including the visceral and parietal pericardium. The visceral layer is quite thin and is closely applied to the epicardium, great vessels, superior and inferior vena cava, and pulmonary arteries and veins. The outer parietal layer is much thicker and is composed primarily of collagen. The parietal layer is attached to the manubrium and xiphoid portions of the sternum, the diaphragm, and the vertebral column. These attachments stabilize the heart within the thorax. The accumulation of fluid within these spaces can result in cardiac compression, reduction in venous return, and ultimately tamponade depending upon pericardial distensibility. Cardiac tamponade is a medical emergency and requires immediate fluid resuscitation, inotropic support, vasopressor support and ultimately mechanical drainage of the fluid to alleviate the primary physiologic derangement- impaired diastolic filling.

Acute Type-A aortic dissection requires emergency surgery and is associated with considerable mortality. Tears in the intimal layer result in the propagation of the dissection secondary to blood entering the intima-media potential space. Reported mortality remains high despite advancements in diagnostic and therapeutic modalities [1-3]. Although acute aortic dissection classically produces a sudden onset of severe chest pain that often has a tearing or ripping quality, no one sign or symptom can positively identify aortic dissection. The clinical manifestations are diverse, making the diagnosis difficult and requiring a high clinical index of suspicion. Accordingly, an estimated $38 \%$ of acute aortic dissections are missed on initial evaluation [4-6].

\section{Case Report}

An 84-year-old female presented after a fall that was preceded by an episode of transient chest pain. Upon arrival to the emergency department, the patient was somnolent, hypotensive $(80 / 40 \mathrm{mmHg})$ and tachycardic (150 bpm). Physical examination revealed a $15 \mathrm{mmHg}$ pulsus paradoxus, muffled cardiac sounds, elevated jugular venous pressure and lung crackles. No auscultatory findings consistent with aortic insufficiency were noted. Initial laboratory evaluation revealed metabolic acidosis, anemia, and normal renal function. No other pertinent findings were available at the time.

The 12-lead electrocardiogram revealed sinus tachycardia without ST-segment elevation. Chest X-ray showed no mediastinal widening or pleural effusion. Initial troponin I was only minimally elevated at $0.8 / \mathrm{ng} / \mathrm{ml}$. An initial echocardiogram showed normal left ventricular (LV) function, reduced right ventricular function, and a large localized pericardial effusion with apical adherent hematoma (Figure 1a). There was no evidence of proximal aortic dissection flap, but suggestion of contained apical LV wall rupture (Figure $1 \mathrm{~b}$ ). However, an intravenous injection of perflutren protein-type A microspheres $\left(\mathrm{Optison}^{\mathrm{Tm}}\right.$ ) revealed no flow through the putative site of myocardial rupture (Figure 1c). There was echocardiographic evidence of hemodynamic compromise including right ventricle diastolic collapse, as well as significant (>30\%) tricuspid and mitral inflow pattern variations with respiration.

The patient became rapidly unstable with refractory hypotension and respiratory failure requiring intubation, volume resuscitation, increasing vasopressors; an emergency pericardial window was therefore performed at the bedside. Ongoing manual aspiration of frank pericardial blood was performed with a staccato pattern of hemodynamic improvement, followed by re-bleeding and hypotension. Given continuous bleeding into the pericardium the decision was made to perform an emergency exploration of the pericardium, ventricular apex and proximal aorta. There was no evidence of LV apical wall perforation. On inspection, a Type A aortic dissection had perforated into the pericardial space involving the aortic valve. Attempts to

*Corresponding author: Mori J Krantz, Denver Health Medical Center, 777 Bannock St. MC 0960, Denver CO 80204, USA, Tel: (303) 436-7818; Fax: (303) 437-7739; E- mail: Mori.Krantz@dhha.org

Received October 24, 2013; Accepted January 13, 2014; Published January 20 2014

Citation: Garcia JA, Krantz MJ (2014) Refractory Tamponade: Use of Ultrasound Contrast Agent to Distinguish Aortic Dissection from Cardiorrhexis. J Cardiovasc Dis Diagn 2: 139. doi:10.4172/2329-9517.1000139

Copyright: $\odot 2014$ Garcia JA, et al. This is an open-access article distributed unde the terms of the Creative Commons Attribution License, which permits unrestricted use, distribution, and reproduction in any medium, provided the original author and source are credited. 
Citation: Garcia JA, Krantz MJ (2014) Refractory Tamponade: Use of Ultrasound Contrast Agent to Distinguish Aortic Dissection from Cardiorrhexis. J Cardiovasc Dis Diagn 2: 139. doi:10.4172/2329-9517.1000139

Page 2 of 3

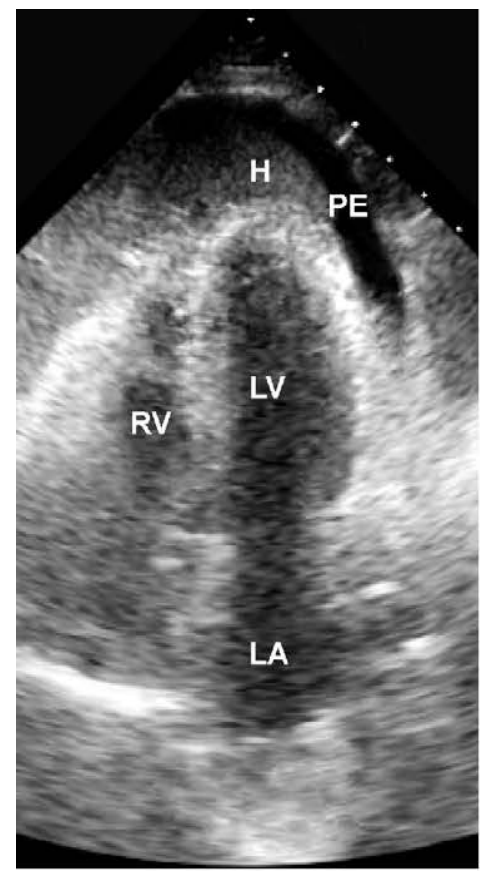

Figure 1a: This apical 4-chamber view shows a large pericardial effusion with a hypoechoic organized appearance likely consistent with hematoma/ thrombus $(\mathrm{H})$ and a pericardial effusion $(\mathrm{PE})$.

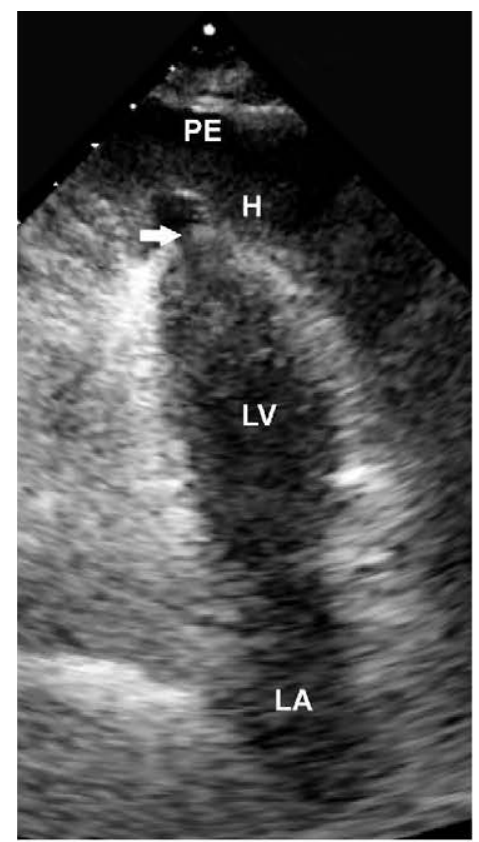

Figure 1b: Findings suggestive of apical perforation (white arrow) with pericardial hematoma/thrombus formation $(H)$.

repair the dissection were complicated by refractory hypotension with electrical instability and the patient ultimately expired.

\section{Discussion}

Cardiac tamponade carries a significant mortality rate whether related to cardiorrhexis or aortic dissection [3,7]. Exclusion of cardiac rupture using ultrasound enhanced contrast echocardiography was helpful in defining a preoperative diagnosis of aortic dissection. Tamponade complicating an aortic dissection is one of the most common causes of death from aortic dissection. Some reports show an early mortality as high as $60 \%$ [8].

The present case illustrated the presence of Becks triad (hypotension, muffled heart tones and jugular venous distension). This triad was first described by the thoracic surgeon Claude Beck in the setting of penetrating trauma. In such cases the patient is unable to fully compensate for the sudden impairment to diastolic filling and blood pressure falls. In contrast, in the absence of trauma tamponade often develops more gradually, and blood pressure is frequently preserved [9]. In addition the presence of tachycardia, pulsus paradoxus and right heart collapse observed on echocardiography corroborated the diagnosis.

Given the history and presentation diagnostic consideration must include both cardiorrhexis and aortic dissection. The electrocardiogram and chest X-ray were not suggestive of either acute myocardial infarction or aortic dissection respectively. Moreover, there was no auscultatory evidence of aortic insufficiency or pulse discrepancy between the upper extremities. Further complicating matters, there was no echocardiographic evidence of dissection flap or aortic valve involvement. The clinical confounder was the echo-lucent space suggestive of LV wall perforation. Despite no flow communication this introduced diagnostic uncertainty and potential surgical delay that was obviated by the use of ultrasound contrast agent. This finding coupled with the absence of recent myocardial infarction and negative cardiac biomarkers excluded this diagnosis. The patients continued hemodynamic instability and refractory hypotension precluded further evaluation and an emergency pericardial window was performed at the bedside. Two things became rapidly obvious after the pericardial window: 1) there was no evidence of cardiorrhexis or flow from the apex and 2) there was active bleeding into the pericardial space with

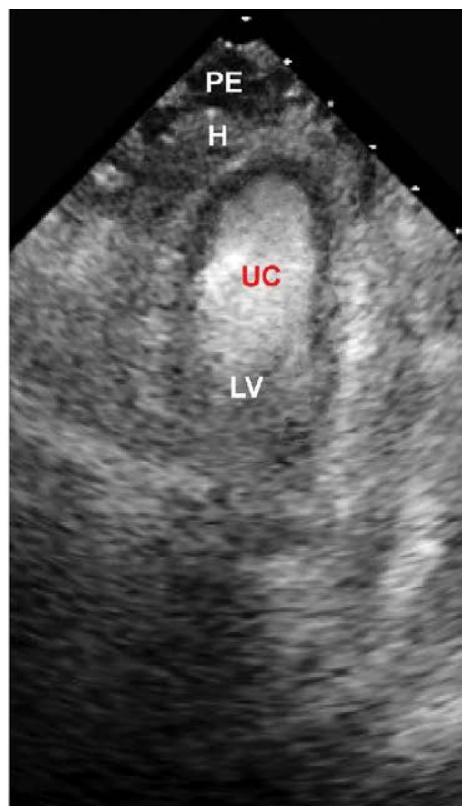

Figure 1c: Ultrasound contrast (UC) with protein-type A microspheres (Optison ${ }^{\circledR}$ ) revealing no flow from the left ventricle (LV) to the pericardium. There is now a clear definition of the endocardium. 
worsening hemodynamic instability. These factors suggest that the drainage of pericardial fluid resulted in worsening hemodynamics as the intra-pericardial pressure likely acted as a "stop-valve" which inhibited the bleeding source.

Well defined management of acute aortic dissection exists; however, little is known about optimal management of hemopericardium that complicates the most severe cases [8]. This case highlights the fact that the medically necessary pericardial window may have paradoxically worsened hemodynamics. Isselbacher et al. reported a series of patients with tamponade and proximal aortic dissection [8]. Three of the 10 presented with sudden onset of fatal electromechanical dissociation, 6 presented with hypotension, and 1 was normotensive on presentation. Of the 7 hypotensive or normotensive patient diagnosed with cardiac tamponade 4 underwent successful pericardiocentesis, while awaiting surgery. At time intervals of 5-40 minutes after the pericardiocentesis, 3 of the 4 patients experienced sudden onset of electromechanical dissociation and death; the fourth patient survived and underwent surgical repair. It is important to note that while $3 / 10$ patients died from electromechanical dissociation immediately upon presentation, the 3 other deaths occurred immediately after successful pericardiocentesis, a procedure intended to stabilize them. This suggests that pericardiocentesis in these patients may not necessarily result in a clinical benefit. Although this is an observation based upon a very small sample size, this is the largest series describing aortic dissection with tamponade and the impact of a pericardiocentesis. It is also important to note that a Type A aortic dissection and a stable ventricular rupture require immediate surgical correction. In that case the role of echocardiography would be to simply provide better guidance to the potential repair techniques and extent of involvement. The present case illustrates an important caution to clinicians regarding the risk of pericardial drainage in acute aortic dissection. It also emphasizes the potential for ultrasound enhanced contrast echocardiography to rapidly exclude the presence of cardiorrhexis. To our knowledge this is the first documented case where ultrasound contrast was used to differentiate cardiorrhexis from aortic dissection.

\section{References}

1. Hagan PG, Nienaber CA, Isselbacher EM, Bruckman D, Karavite DJ, et al (2000) The International Registry of Acute Aortic Dissection (IRAD): new insights into an old disease. JAMA 283: 897-903.

2. Patel PD, Arora RR (2008) Pathophysiology, diagnosis, and management of aortic dissection. Ther Adv Cardiovasc Dis 2: 439-468.

3. Guberman BA, Fowler NO, Engel PJ, Gueron M, Allen JM (1981) Cardiac tamponade in medical patients. Circulation 64: 633-640.

4. Sutherland A, Escano J, Coon TP (2008) D-dimer as the sole screening test for acute aortic dissection: a review of the literature. Ann Emerg Med 52: 339-343.

5. Spittell PC, Spittell JA Jr, Joyce JW, Tajik AJ, Edwards WD, et al. (1993) Clinical features and differential diagnosis of aortic dissection: experience with 236 cases (1980 through 1990). Mayo Clin Proc 68: 642-651.

6. von Kodolitsch Y, Nienaber CA, Dieckmann C, Schwartz AG, Hofmann T, et al. (2004) Chest radiography for the diagnosis of acute aortic syndrome. Am J Med 116: 73-77.

7. Bayegan K, Domanovits H, Schillinger M, Ehrlich M, Sodeck G, et al. (2001) Acute type $\mathrm{A}$ aortic dissection: the prognostic impact of preoperative cardiac tamponade. Eur J Cardiothorac Surg 20: 1194-1198.

8. Isselbacher EM, Cigarroa JE, Eagle KA (1994) Cardiac tamponade complicating proximal aortic dissection. Is pericardiocentesis harmful? Circulation 90: 23752378.

9. Krantz MJ, Woods JE, Havranek EP, Linas S (2002) Simultaneous malignant hypertension and cardiac tamponade. Am J Kidney Dis 39: E17. 